\title{
A Comparison of the Effects of First Metatarsophalangeal Joint Arthrodesis and Hemiarthroplasty on Function of Foot Forces using Gait Analysis
}

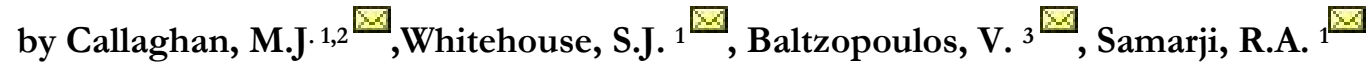

The Foot and Ankle Online Journal 4 (12): 1

Background: Arthroplasty or hemiarthroplasty for hallux rigidus of the first metatarsophalangeal ( $1^{\text {st }}$ MTPJ) is thought to lead to greater joint motion and improved gait function. There have been no studies to compare the effects of $1^{\text {st }} M T P J$ arthrodesis and hemiarthroplasty on joint kinetics, kinematics and plantar pressures of foot and lower limb.

Methods: A retrospective, case series pilot study approximately 22 months post operation. Subjects had either arthrodesis or a NorthStar HemiCAP hemiarthroplasty for osteoarthritis of the $1^{\text {st }}$ MTPJ. All had plantar pressure, kinetic and kinematic gait analysis as well as the Foot and Ankle Outcome Score (FAOS), and $1^{\text {st }}$ MTPJ range of motion (ROM).

Results: For the hemiarthroplasty subjects the mean ROM on the non-operated $1^{\text {st }}$ MTPJ was $49^{0}$ (SD23) but only19 (SD16) on the operated side. Kinetic and kinematic results for both operations were similar. Plantar peak pressure data showed that the arthrodesis group took more pressure under the $1^{\text {st }}$ MTPJ in the stance phase. The arthrodesis group had higher FAOS scores (mean 95.6 SD 5.1) than the hemiarthroplasty group (mean 72.2 SD 18.8) indicating more satisfaction with their surgery.

Conclusions: Arthrodesis of the $1^{\text {st }}$ MTPJ better FOAS scores, improved peak plantar pressure over the medial foot compared to a $1^{\text {st }}$ MTPJ hemiarthroplasty. There were minimal differences in kinetic and kinematic data. Hemiarthroplasty patients had considerably reduced $1^{\text {st }}$ MTPJ ROM compared to their non-operated side. We conclude that the North Star HemiCAP cannot be recommended at this time for the management of hallux rigidus. Arthrodesis remains the surgical treatment of choice.

Key Words: Monofilament, tuning fork, ankle reflex, superficial pain, neuropathy.

Accepted: November, 2011

Published: December, 2011

This is an Open Access article distributed under the terms of the Creative Commons Attribution License. It permits unrestricted use, distribution, and reproduction in any medium, provided the original work is properly cited. @The Foot and Ankle Online Journal (www.faoj.org), 2011 All rights reserved.

$\mathrm{F}$ irst metatarsophalangeal joint (MTPJ) arthritis is a progressive, painful and debilitating condition also known as hallux rigidus. ${ }^{1}$ The most common form of presentation is unilateral involvement in the older female patient with a gradual onset of pain and limited range of motion at the $1^{\text {st }}$ MTPJ.

Address correspondence to: Michael Callighan, Manchester Royal Infirmary, Manchester,UK. E-mail: michael.callaghan@manchester.ac.uk

${ }^{1}$ Manchester Royal Infirmary, Grafton Street, Manchester, UK.

${ }^{2}$ University of Manchester, Oxford Road Manchester, UK.

${ }^{3}$ Manchester Metropolitan University, John Dalton Building Manchester, UK.
This often results in an abnormal gait pattern due to a shift in weight bearing from the first ray to the outer border of the foot ${ }^{11}$ along the metatarsal heads transverse axis.

In a recent study on hallux rigidus ${ }^{5} 90 \%$ of patients considered that their walking pattern had changed of which $33 \%$ considered that this change affected their everyday lives. Only 51 feet $(28 \%)$ were able to push through the ground at propulsion, the remainder was affected to varying degrees of severity.

(C) The Foot and Ankle Online Journal, 2011 
One hundred and thirty five feet (75\%) rolled outwards during the propulsion phase of the gait cycle and it is claimed that these changes may be detrimental to a person's gait.

Surgical treatments for hallux rigidus include arthrodesis, cheilectomy, excisional arthroplasty, metatarsal osteotomy, and total or hemiarthroplasty. Of these techniques, arthrodesis of the $1^{\text {st }}$ MTPJ is regarded currently as the mainstay of surgical treatment ${ }^{15}$, and is still considered the gold standard particularly in younger and more active patients. The technique is advocated for the alleviation of pain and deformity and restoration of the stability and weight bearing function of the first ray by stabilizing the medial column of the foot. ${ }^{3,10}$ This technique has been shown to be successful both in pain relief and also in clinical and radiographic evaluation.' Despite the high patient satisfaction in terms of pain relief, $1^{\text {st }}$ MTPJ arthrodesis has been shown to reduce functional gait parameters compared to the contralateral limb. For example, DeFrino et al., ${ }^{6}$ using dynamic pedobarography on 10 feet, showed that $1^{\text {st }}$ MTPJ arthrodesis produced a restoration of the weight bearing function of the first ray with greater maximum force carried by the distal hallux at toe-off. However, the same study also showed a statistically significant reduction in ankle torque and ankle power at push-off and a shorter step length and loss of ankle plantar flexion at toe-off. Similarly, Brodsky, et al., ${ }^{3}$ compared pre and post operative arthrodesis data and found three statistically significant changes in gait: an increased single limb support time, a decrease in step length but an increase in maximum ankle push off power in contrast to the previous study. Although these finding were attributed to improvements in pain and mechanical stability, mechanics of the $1^{\text {st }}$ MTPJ were not measured.

Stiffness resulting from an arthrodesis can lead to patient dissatisfaction with the arthrodesis technique. Normal gait patterns can be altered, with a decreased step length and some loss of ankle plantar flexion at toe-off on the fused side. ${ }^{6}$ Additionally, the long term effects of a rigid first ray may not yet be apparent. ${ }^{9}$
An alternative and logical follow up is first MTPJ arthroplasty. Whether using a total or hemiarthroplasty, the aims of joint replacement are not only to provide pain relief but also restore motion of the first MTPJ, maintain joint stability with a concomitant improvement in function and gait. $^{12}$ Many different designs and solutions for arthroplasty have been proposed over time.

Evidence to support the increase in range of movement and improved gait is scanty and to date there has only been one randomized, controlled trial comparing arthrodesis to arthroplasty for hallux rigidus. ' This study compared 38 toes undergoing arthrodesis with 39 undergoing a Biomet unconstrained total arthroplasty. Pain was measured via a visual analogue scale (VAS) as well as patient self reported satisfaction, first MTPJ mobility using electrogoniometry and plantar foot pressures using pedobarography. The results showed that the arthrodesis group was statistically better in pain at 12 $(p=0.005)$ and 24 months $(p=0.01)$. First MTPJ range of motion in the arthroplasty group improved post operatively compared to the pre operative status, but not to a level of statistical significance. Gait analysis on an 8 meter walkway measured plantar foot pressures and showed that patients in both groups tended to weight bear on the lateral border of the foot. In the arthrodesis group, all fusion sites were united and had gained a significant improvement in pain levels. The authors were not in favor of total arthroplasty as several implants had to be removed due to loosening. They were not able to assess the effects of either procedures on other joints of the foot and ankle during walking due to the lack of kinetic and kinematic analysis.

A recent non-randomized retrospective study ${ }^{13}$ compared the BioPro metallic hemiarthroplasty in 21 toes to first MTPJ arthrodesis in 27 toes and at 6 year follow up found inferior clinical and functional results in the arthroplasty group as assessed by the AOFAS Hallux Metatarsal Interphalangeal (AOFAS-HMI) score. There was no gait analysis of any kind performed. 


\begin{tabular}{|l|l|l|l|l|l|l|}
\hline & $\begin{array}{l}\text { Age } \\
\text { (SD) }\end{array}$ & $\begin{array}{l}\text { Gender } \\
\text { M:F }\end{array}$ & $\begin{array}{l}\text { Weight } \\
\text { Kg(SD) }\end{array}$ & $\begin{array}{l}\text { Height } \\
\text { Cm (SD) }\end{array}$ & $\begin{array}{l}\text { Side } \\
\text { L:R }\end{array}$ & Time since operation (months)(SD) \\
\hline Arthrodesis & $\begin{array}{l}58.2 \\
(15)\end{array}$ & $1: 4$ & $70(7)$ & $164(8)$ & $1: 4$ & 23.7 \\
$(5.9)$ \\
\hline $\begin{array}{l}\text { Hemi- } \\
\text { arthroplasty }\end{array}$ & $\begin{array}{l}60.5 \\
(7)\end{array}$ & $0: 4$ & $68(6)$ & $159(3)$ & $2: 2$ & 22.3 \\
\hline
\end{tabular}

Table 1 Descriptive statistics of subjects.

Despite the theory that an arthroplasty would lead to greater first MTPJ motion and an improvement in gait function, there have been no studies to compare the effects of first MTPJ arthrodesis and hemiarthroplasty on both the joint kinetics and kinematics of foot and lower limb using gait analysis. These techniques combined in a specialist gait laboratory form a powerful and robust analysis of gait, joint mobility and muscle function. They would help our understanding of both procedures by providing information to assess the effects of each procedure on the other joints of the forefoot and gait mechanics and function in general, which are important for the long term prognosis of these techniques. We have identified some methodological issues arising from the studies cited: Firstly, there has been no gait analysis comparison of hemiarthroplasty with arthrodesis of the first MTPJ and their effects on foot and ankle kinetics and kinematics. Secondly, there has been no detailed analysis comparing the plantar pressure distribution of arthrodesis and hemiarthroplasty.

Therefore, the aims of this study were to observe any differences in kinematic, kinetic and plantar pressure measurements between first MTPJ hemiarthroplasty and first MTPJ arthrodesis. The study design took the form of a pilot study to ascertain technical feasibility and also to provide data exploring clinical significance and sample size for a future randomized controlled surgical trial.

\section{Methods}

This was a retrospective, case series pilot study.

\section{Subjects}

All patients were identified from the patient operating list of the senior surgeon (RAS) and had surgery for non- inflammatory, symptomatic hallux rigidus. There were five patients ( 4 females) with first MTPJ arthrodesis (Age $58.2 \pm 15$ years, mass $70 \pm 7 \mathrm{~kg}$ and height $164 \pm 8 \mathrm{~cm})$ and four female patients with first MTPJ hemiarthroplasty (Age $60.5 \pm 7$ years, mass $68 \pm 6 \mathrm{~kg}$, height $159 \pm 3 \mathrm{~cm})$. Descriptive statistics are listed in Table 1. The mean post operation time was 23 months after arthrodesis and 22 months after hemiarthroplasty. None of the subjects had experienced any post-operative complications such as superficial or deep infection, deep venous thrombosis, or complex regional pain syndrome. There were no cases of non-union or component loosening and none of the arthrodeses or hemiarthroplasties had been revised.

\section{Exclusion Criteria}

Subjects were excluded from the study if they had undergone other foot and ankle surgery, other degenerative joint diseases of the lower limb, post infectious arthritis, rheumatoid arthritis or Charcot neuroarthropathy. 


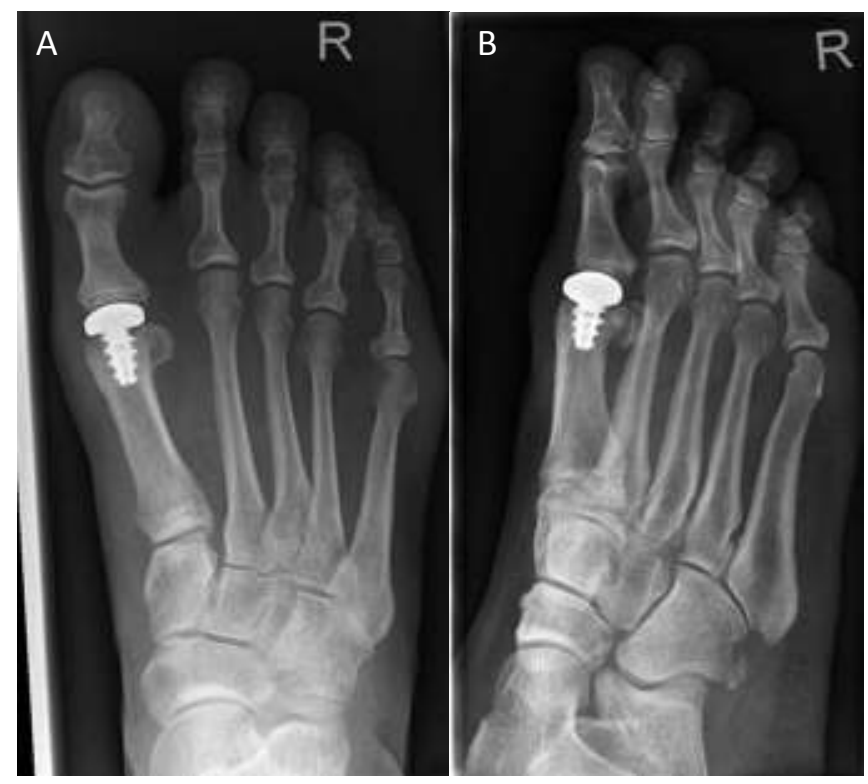

Figure 1A and 1B Plain radiographs anterior posterior (A) and oblique (B) views of the NorthStar HemiCAP prosthesis.

\section{Surgical procedures}

\section{First MTPJ hemiarthroplasty}

A NorthStar HemiCAP implant MTP resurfacing implant was used for all hemiarthroplasty patients. (Figure 1A and 1B) First, a dorsal approach to the first MTPJ was used to expose the joint. A guide pin was inserted into the first metatarsal head via the distal articular surface using a drill guide to aid location of the correct axis. A cannulated drill was then used to drill over the guide wire. The hole was tapped and a taper post inserted. A trial cap was placed on the taper post to determine whether the correct depth has been achieved. The trial cap was removed and a centering shaft and contact probe inserted to determine offsets at four index points. The articular component size was determined from a sizing card using these offset values. A circle cutter was used over the guide pin to score the articular cartilage down to subchondral bone. A surface reamer, size matched to the chosen articular component was used next. A final sizing trial was inserted and any necessary adjustments made. Finally, the actual articular components was inserted and impacted down.

(C) The Foot and Ankle Online Journal, 2011

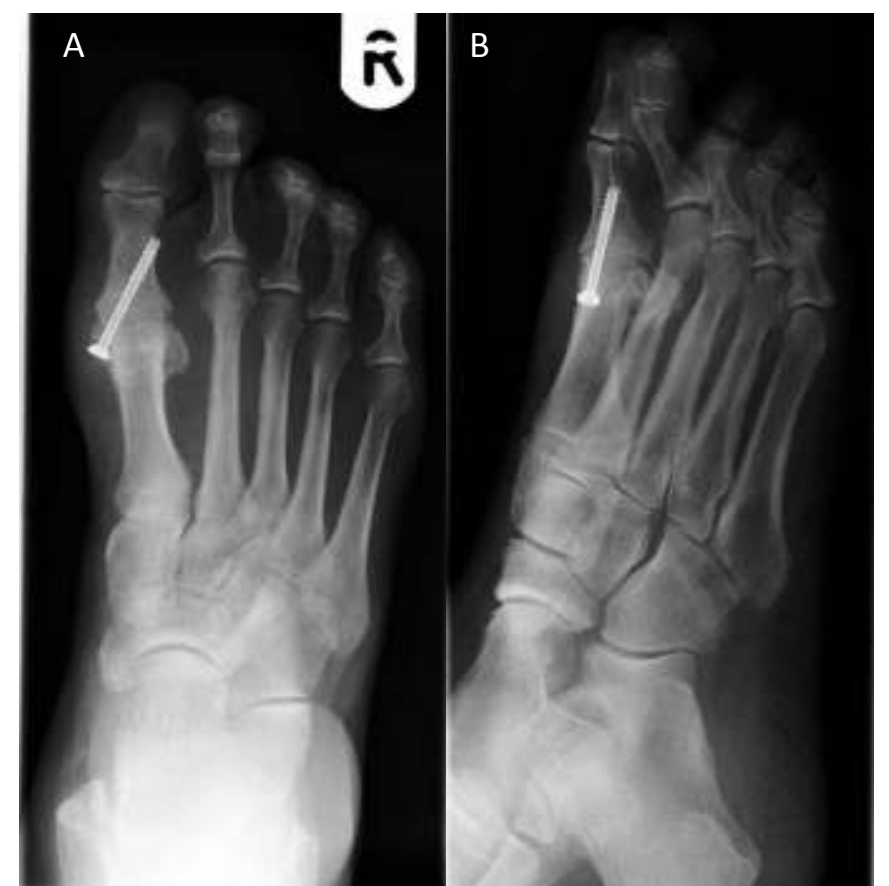

Figure 2A and 2B Plain radiograph anterior posterior $(A)$ and oblique (B) views of the arthrodesis technique.

Post operatively, no plaster cast was applied. Patients were encouraged to fully weight bear as soon as possible without walking aides. In particular, a normal gait pattern was permitted in order to achieve dorsiflexion at the MTPJ at the push off phase of the gait cycle. A check radiograph was requested 6 week post operatively.

\section{First MTPJ Arthrodesis}

The first MTPJ arthrodesis was carried out under tourniquet through a dorsal longitudinal incision centered over the metatarsophalangeal joint. (Figure $2 \mathrm{~A}$ and $2 \mathrm{~B}$ ) The dorsal sensory nerve was seen and protected throughout the procedure. A capsulotomy was carried out and the metatarsal head and proximal phalanx were denuded of articular cartilage using reamers to expose bleeding cancellous bone. They were coapted and held with a partially threaded cancellous small fragment screw with a washer. The toe was positioned taking reference from the heel with slight dorsiflexion at the MTPJ in a neutral rotation and slight valgus position. The wound was closed in layers. 
Post operatively, patients were required to strictly heel weight bear with the help of walking aides for 6 weeks. A plaster slab was put over the operation site, but did not include the ankle or lateral border of the foot. Graduated heel weight bearing and discarding of the walking aides was permitted after a check radiograph 6 weeks post operation.

\section{Outcome Measures}

\section{The foot and ankle outcome score (FAOS)}

The FAOS ${ }^{14}$ is a patient administered score consisting of 5 subscales; i) pain, ii) other Symptoms, iii) function in daily living (ADL), iv) function in sport and recreation (Sport/Rec), and v) foot and anklerelated Quality of Life (QOL). The last week was taken into consideration when answering the questionnaire. Standardized answer options were given (5 Likert boxes) and each question got a score from 0 to 4. A normalized score (100 indicating no symptoms and 0 indicating extreme symptoms) was calculated for each subscale. The results were plotted as an outcome profile.

\section{MTPJ Range of motion}

A small finger universal goniometer with five degree increments was used to measure first MTPJ range of motion (ROM) in dorsiflexion and plantarflexion. This was done in supine lying with the heel resting on the examination couch and the ankle in a neutral position.

\section{Gait analysis}

Gait analysis was performed on a 10 meter m instrumented walkway at the gait analysis laboratory at Manchester Metropolitan University. Kinematic and kinetic measurements and plantar pressures are affected by walking speed so participants were instructed to walk at their normal comfortable speed after familiarization with the testing procedures.

Kinematic data were acquired using a threedimensional (3D) optoelectronic motion analysis system (VICON Nexus, Oxford Metrics, Oxford, England) with ten high-resolution cameras sampling at $120 \mathrm{~Hz}$.
The kinematic data was filtered with cross-validating quintic splines interpolation (QSI scheme) to reduce the noise from skin movement and other sources of coordinate recording errors. The standard biomechanical foot model used in typical gait analysis applications represents the foot as a single rigid body, allowing the assessment of only foot progression angle and net dorsi/plantar flexion. This is not adequate for the purpose of this study and the examination of the MTPJ function. For this reason we used the "Oxford Foot model" ${ }^{4}$ which represented the foot using three rigid segments (hindfoot, forefoot and hallux). The relative motion between the segments was described without intersegment constraints in this foot model. This was necessary to accommodate the likely conditions and constraints of hallux and forefoot function in the patients of this study. For the hindfoot, segment markers were placed on the lateral and medial malleolus two markers along the vertical axis of the calcaneus posteriorly and two additional markers on the lateral and medial sides of the calcaneus. For the Forefoot and Hallux segments markers were placed on the distal medial side of the $1^{\text {st }}$ MTPJ, the distal lateral side of the $5^{\text {th }}$ metatarsal joint, on the distal $2^{\text {nd }}$ MTPJ, on the proximal $1^{\text {st }}$ and $5^{\text {th }}$ MTPJ, and two markers (proximal and distal) on the long axis of the hallux. For the other leg segments the standard Helen Hayes marker set was used as implemented in the Bodybuilder modeling package of the VICON system. Static trials were performed before the gait tests to allow the calculation of segment-embedded coordinate frames based on the positions of the anatomical landmarks that were identified from the coordinates of the surface markers and relevant subject anthropometric measurements. Kinematic parameters to include joint angles and kinetic measures including joint moments and plantar pressures were calculated at key moments in the stance phase of gait cycle (heel strike, foot flat, midstance, heel off and toe off) for comparisons between the operated and unaffected side of the two different groups of patients.

Kinetic (ground reaction force) data were collected at $1080 \mathrm{~Hz}$ using two Kistler force-plates (Kistler, Switzerland) that are embedded in the middle of the walkway. 


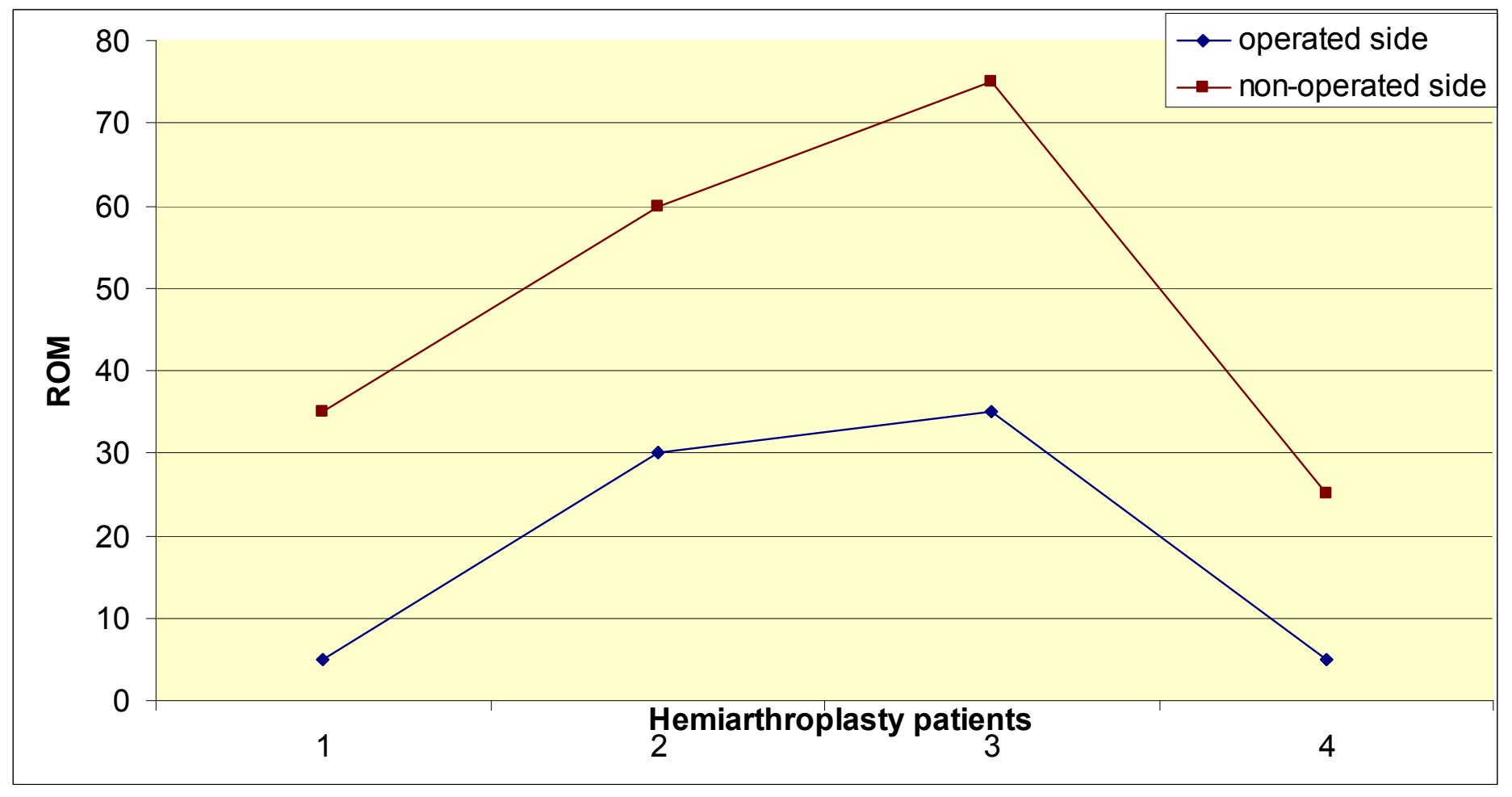

Figure 3 Range of motion (ROM) at $1^{\text {st }}$ MTPJ compared to non-operated side in hemi -arthroplasty patients.

Joint kinetics (resultant joint forces and moments) were calculated using standard inverse dynamics techniques by combining the kinematic and force plate data into a musculoskeletal mechanical model of the lower limbs. Data were normalized to body weight.

Plantar pressures were recorded during level walking with the participants wearing their own shoes. The Tekscan in-shoe plantar pressure system was used for these measurements (Tekscan, Boston, MA), sampling at a frequency of $40 \mathrm{~Hz}$. The Tekscan Research Foot software was used to construct individual "masks" to determine maximum force $(\mathrm{kg})$ and peak pressure $\left(\mathrm{kg} / \mathrm{cm}^{2}\right)$ under several regions of interest of the foot including the hallux, toes, metatarsals, midfoot and heel.

The pressures in the plantar surface of the foot during normal gait were examined with Tekscan in-shoe plantar pressure system using the F-Scan sensors (Model \#3000E VersaTek). These sensors contain up to 960 individual pressure sensing elements, that which detect the subject's plantar pressure.
These pressure elements are arranged in rows and columns on the sensor and their output recorded by the system during any activity is quantified into 256 increments. This digital value is then calibrated for pressure in kilopascals $(\mathrm{KPa})$. The sensors were cut to size for each subject and were inserted in the shoes so that the pressures exerted between the foot and the shoe were recorded during the gait.

A "step" calibration procedure was followed where the subject is required to shift the weight to each foot in turn so that the full weight of the subject is sensed alternately by both sensors (left and right). According to the instructions of the manufacturer, this is the most appropriate calibration technique for dynamic activities such as gait because it contains a factor for rapid dynamic changes and compensates for time related changes in sensor output. Following the calibration procedure, the subjects were asked to walk in their normal gait speed on a flat gait walkway of approximately 10 meters, three times. The first two trials were used for familiarization with the instrumentation in the shoes and although data were collected from all three trials only the third (final) trial was analyzed. 


\begin{tabular}{|c|c|c|c|c|c|c|c|c|}
\hline & \multicolumn{4}{|c|}{ Operation side } & \multicolumn{4}{|c|}{ Non-operation side } \\
\hline & $\begin{array}{c}\text { Ankle } \\
\text { Max DF } \\
\text { (deg) }\end{array}$ & $\begin{array}{c}1^{\text {st }} \text { MTPJ } \\
\text { RoM } \\
\text { (deg) }\end{array}$ & $\begin{array}{c}\text { Peak Force } \\
\text { (Fx) } \\
\text { \%BW } \\
\end{array}$ & $\begin{array}{c}\text { Ankle } \\
\text { total RoM } \\
\text { (deg) }\end{array}$ & $\begin{array}{c}\text { Ankle } \\
\text { Max DF } \\
\text { (deg) }\end{array}$ & $\begin{array}{c}1^{\text {st }} \text { MTPJ } \\
\text { RoM } \\
\text { (deg) }\end{array}$ & $\begin{array}{l}\text { Peak force } \\
\text { (Fx) \%BW }\end{array}$ & $\begin{array}{l}\text { Ankle total } \\
\text { RoM (deg) }\end{array}$ \\
\hline Arthrodesis & $\begin{array}{l}18.90 \\
(5.43)\end{array}$ & $\begin{array}{c}2.86 \\
(1.12)\end{array}$ & $\begin{array}{c}5.64 \\
(01.26)\end{array}$ & $\begin{array}{l}18.69 \\
(7.10)\end{array}$ & $\begin{array}{l}19.17 \\
(2.02)\end{array}$ & $\begin{array}{l}10.45 \\
(6.55)\end{array}$ & $\begin{array}{c}4.63 \\
(2.66)\end{array}$ & $\begin{array}{l}18.60 \\
(2.70)\end{array}$ \\
\hline $\begin{array}{l}\text { Hemi- } \\
\text { arthroplasty }\end{array}$ & $\begin{array}{l}24.44 \\
(6.21)\end{array}$ & $\begin{array}{c}3.39 \\
(0.19)\end{array}$ & $\begin{array}{c}5.81 \\
(0.68)\end{array}$ & $\begin{array}{l}21.82 \\
(0.46)\end{array}$ & $\begin{array}{l}27.07 \\
(1.46)\end{array}$ & $\begin{array}{l}14.56 \\
(8.41)\end{array}$ & $\begin{array}{c}4.39 \\
(1.57)\end{array}$ & $\begin{array}{l}21.48 \\
(3.25)\end{array}$ \\
\hline
\end{tabular}

Table 2 Kinetic and kinematic data Means and (SD). Legend: Max DF = maximum dorsiflexion, $1^{\text {st }}$ MTPJ RoM = first metatarsophalangeal joint range of motion, $\% \mathrm{BW}=$ percentage body weight.

\section{Statistical Analysis}

The analysis was in two parts. Firstly, there was a within groups comparison of the kinetic, kinematic and plantar pressure parameters at key moments of the gait cycle between the operated and non-operated side of each patient. Secondly, there was a between groups analysis of the same parameters between the arthrodesis and hemiarthroplasty patients. The small sample size precluded the use of formal statistical analysis. Comparison of the other data sets was made by analysis of the descriptive statistics and normalized data.

\section{Results}

There were no adverse events from the gait analysis protocol.

The ROM for the $1^{\text {st }}$ MTPJ measured by goniometry was $19^{0}$ (SD16) for the hemiarthroplasty side were compared to $49^{\circ}(\mathrm{SD} 23)$ on the non-operated side. Figure 3 shows the differences in the post-operative ROM at 22 months.

The kinematic results are for the Oxford foot model maximum dorsiflexion and total ROM angles at the ankle and ROM at the $1^{\text {st }}$ MTPJ. For the hemiarthroplasty patients the ROM angle at the $1^{\text {st }}$ MTPJ was small compared to the non-operated side.
There was some measurable motion on the arthrodesis side which was smaller than the hemiarthroplasty. Reasons for the apparent contradiction of available motion in a fused joint are in the discussion. Ankle maximum ROM for dorsiflexion and total ROM were greater in the hemiarthroplasty side compared to the non-operated side and compared to the arthrodesis subjects. (see Table 2).

The kinetic results from the force plate are for the peak medio-lateral force which is affected mainly by supination and pronation of the foot and ankle during the gait cycle from 'heel strike' to 'toe off. A higher ground reaction force in the lateral direction indicated more weight bearing on the medial side of the foot and the first ray. Compared to the non-operated side, the peak lateral force for both the arthrodesis and hemiarthroplasty sides was similar and slightly higher than the operated side. (see Table 2).

The peak plantar pressures from the $1^{\text {st }}$ MTPJ area are presented in Figure 4. The descriptive statistics are in Table 3. These data have several features. The peak plantar pressure area under the first metatarsal was higher on the arthrodesis side compared to the nonoperated side (305 (SD $143 \mathrm{KPa})$ v 194 (SD $73 \mathrm{KPa})$ ) indicating that this part of the foot was taking more pressure than those patients who had a hemiarthroplasty. 


\begin{tabular}{|l|l|l|l|l|}
\hline & Arthrodesis foot & Non-operated foot & Hemiarthroplasty foot & $\begin{array}{l}\text { Non-operated } \\
\text { foot }\end{array}$ \\
\hline Total Peak pressure & $521(153)$ & $419(69)$ & $469(120)$ & $410(148)$ \\
\hline Medial Heel & $316(71)$ & $261(81)$ & $294(47)$ & $340(88)$ \\
\hline Lateral Heel & $292(41)$ & $331(82)$ & $228(84)$ & $276(93)$ \\
\hline Midfoot & $160(104)$ & $122(62)$ & $229(64)$ & $114(44)$ \\
\hline First metatarsal & $305(143)$ & $267(160)$ & $194(73)$ & $215(43)$ \\
\hline Second metatarsal & $383(200)$ & $307(130)$ & $299(85)$ & $248(60)$ \\
\hline Third metatarsal & $410(160)$ & $362(114)$ & $340(100)$ & $282(119)$ \\
\hline Fourth metatarsal & $350(19)$ & $260(67)$ & $367(162)$ & $280(210)$ \\
\hline Fifth metatarsal & $293(151)$ & $149(67)$ & $283(77)$ & $166(85)$ \\
\hline First Toe & $231(104)$ & $239(84)$ & $153(126)$ & $212(130)$ \\
\hline Second Toe & $181(56)$ & $163(86)$ & $132(77)$ & $104(32)$ \\
\hline Third Toe & $112(53)$ & $85(28)$ & $100(74)$ & $65(28)$ \\
\hline Fourth and Fifth Toes & $64(58)$ & $49(37)$ & $43(27)$ & $42(29)$ \\
\hline
\end{tabular}

Table 3 Plantar Peak Pressure data for arthrodesis, hemiarthroplasty and non-operated foot. kPa means (SD).

Furthermore, Table 3 shows that there was a tendency to take greater pressure on the $3 \mathrm{rd}$, 4th, 5th metatarsal heads and the lateral side of the foot in the hemiarthroplasty patients. These data are illustrated in Figure 5 which shows a comparison between left side (normal) and right MTPJ arthrodesis and in Figure 6 which compares left (normal) and right MTPJ hemiarthroplasty. The differences between the plantar pressures at the first metatarsal area were difficult to assess through formal statistical analysis due to the small sample size.

The FAOS scores are presented in Figure 7. These show that the arthrodesis patients (mean 95.6 (SD 5.1) scored higher in most of the subscales and on the overall score than those who had the hemiarthroplasty, (mean 72.2 (SD 18.8)

\section{Discussion}

This study aimed to compare a small group of subjects who had undergone arthrodesis or hemiarthroplasty using the NorthStar HemiCAP prosthesis for hallux rigidus using several gait parameters. Our study had volunteers whose mean post operation time was at least 22 months, thus giving a good representation of the long term outcome of both surgical techniques.
Arthrodesis is generally considered a satisfactory operation for primary OA of the $1^{\text {st }}$ MTPJ. The functional excellence and durability of the results obtained are impressive, although the loss of $1^{\text {st }}$ MTPJ range of motion is an obvious drawback of the technique. ${ }^{7}$ The value of prosthetic implants instead is still a theme for debate. The advantages of the procedure include preservation of the motion and a wide variety of implants have been proposed over time. $^{8}$ Nevertheless high risk of failure of the implant and serious complications have been reported. ${ }^{6}$ The aims of this study were to compare one surgeon experienced in both techniques, to assess clinical efficacy and to use gait analysis methods to help explain any differences between the two operations. The kinematic results indicated that hemiarthroplasty gave only a minimally improved ROM at the hallux, although there was greater movement for maximum ankle dorsiflexion. The discrepancy concerning detectable movement at the fused MTPJ is most probably due to movement of the skin and the markers used for the kinematic analysis relative to the bones.

The kinetic results showed that both techniques resulted in similar laterally directed forces in mid stance. 


\section{1st MTPJ Area Peak Pressure}

operated non-operated

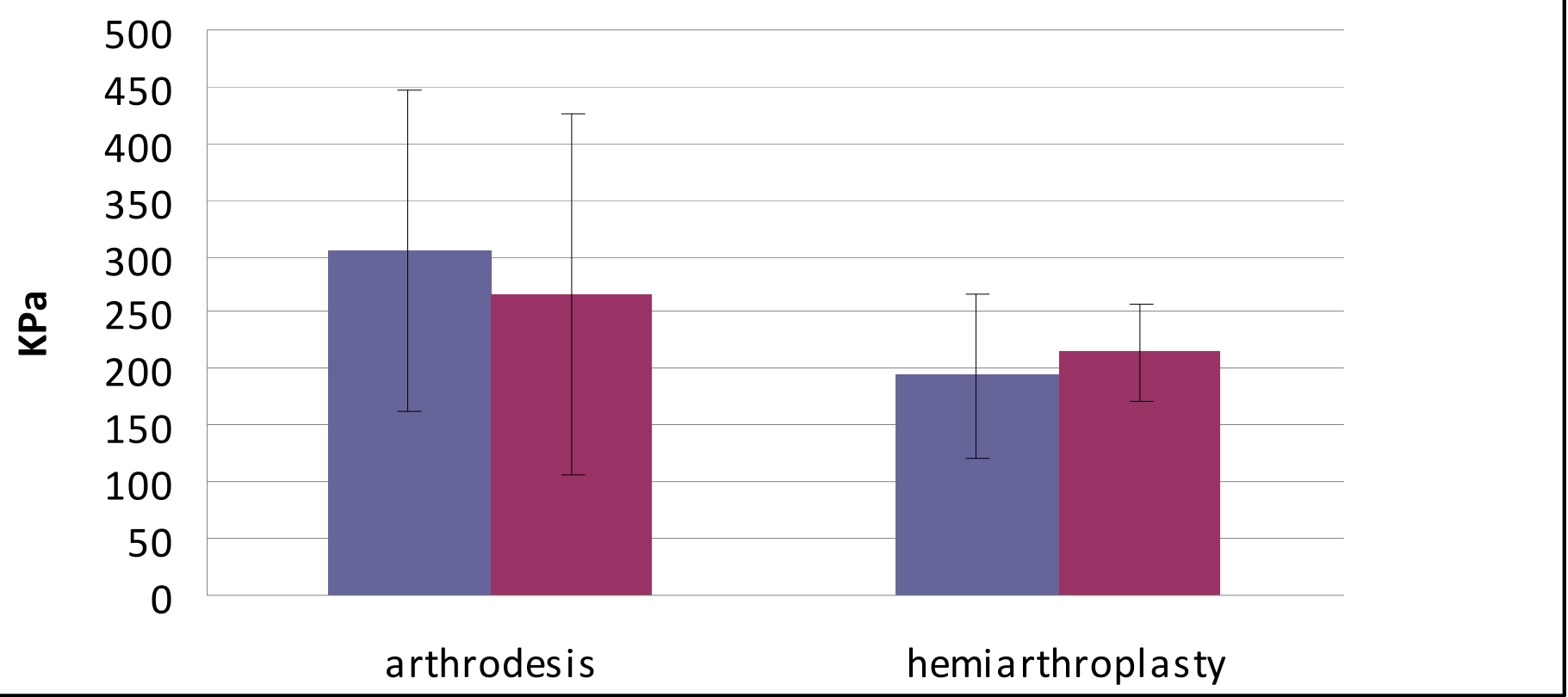

Figure 4 Mean Peak area plantar pressures under the first metatarsal head.

These data indicate that after both arthrodesis and hemiarthroplasty more force can be taken on the inside of the foot compared to the non-operated side. This is in contrast to the observations from the more detailed plantar pressure data which show increased medial foot and $1^{\text {st }}$ MTPJ pressure after the arthrodesis, but not after hemiarthroplasty. It is tempting to infer that this was due to them having less pain at the fused joint and therefore having few problems assuming a normal gait.

This is the first study to use insole sensors to compare plantar pressures of arthrodesis and hemiarthroplasty of the $1^{\text {st }}$ MTPJ. The plantar pressure results showed that the arthrodesis patients were adopting a gait which favored normal $1^{\text {st }}$ MTPJ pressures, whereas the hemiarthroplasty patients, even 22 months post operation, were still favoring the lateral side toes and avoiding pressure under the $1^{\text {st }}$ MTPJ.
These data also show the advantages of plantar pressure and the limitations of ground reaction force analysis in the context of this investigation. Plantar pressure techniques are able to capture more precisely the focal points of pressure distribution (see Figures 5 and 6) whereas force plate readings present a more generalized indication of the overall ground reaction forces generated during gait.

The FAOS shows that all the patients who had hemiarthroplasty were not satisfied with their operation and had continuing pain and stiffness in their toes and foot. Interestingly, only one patient who had arthrodesis considered that foot stiffness was a problem. Four out of five arthrodesis patients considered themselves pain free (i.e.) scoring 'normal' on the pain subscale) whereas only one in four of the hemiarthroplasty group considered themselves pain free. 


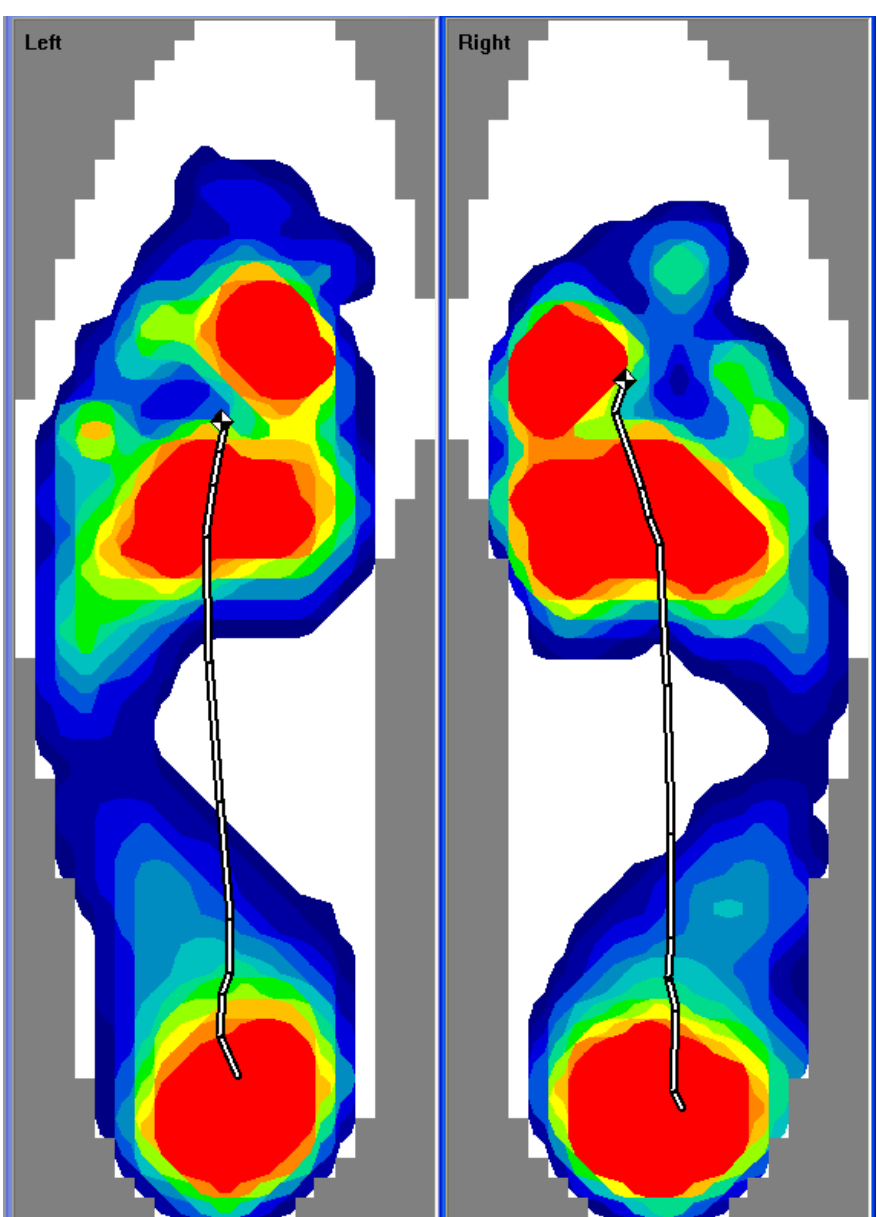

Figure 5 Average peak stance data with center of force trajectory. Patient was 24 months post right arthrodesis.

These results are similar to the data from Raikin, et al., ${ }^{13}$ comparing $1^{\text {st }}$ MTPJ arthrodesis with the BioPro metallic implant. Post-operative scores (using the invalidated American Orthopaedic Foot and Ankle Society Hallux Metatarsophalangeal Interphalangeal AOFAS-HMI scoring system) of 93 for arthrodesis and 71.8 for hemiarthroplasty are very similar to our FAOS of 95.6 and 72.5 respectively. These two sets of results suggest that arthrodesis is superior to hemiarthroplasty in terms of patient self reporting of pain, function and stiffness. This is also the conclusion of systematic review which concluded that arthrodesis achieved better functional outcomes than hemiarthroplasty. ${ }^{2}$

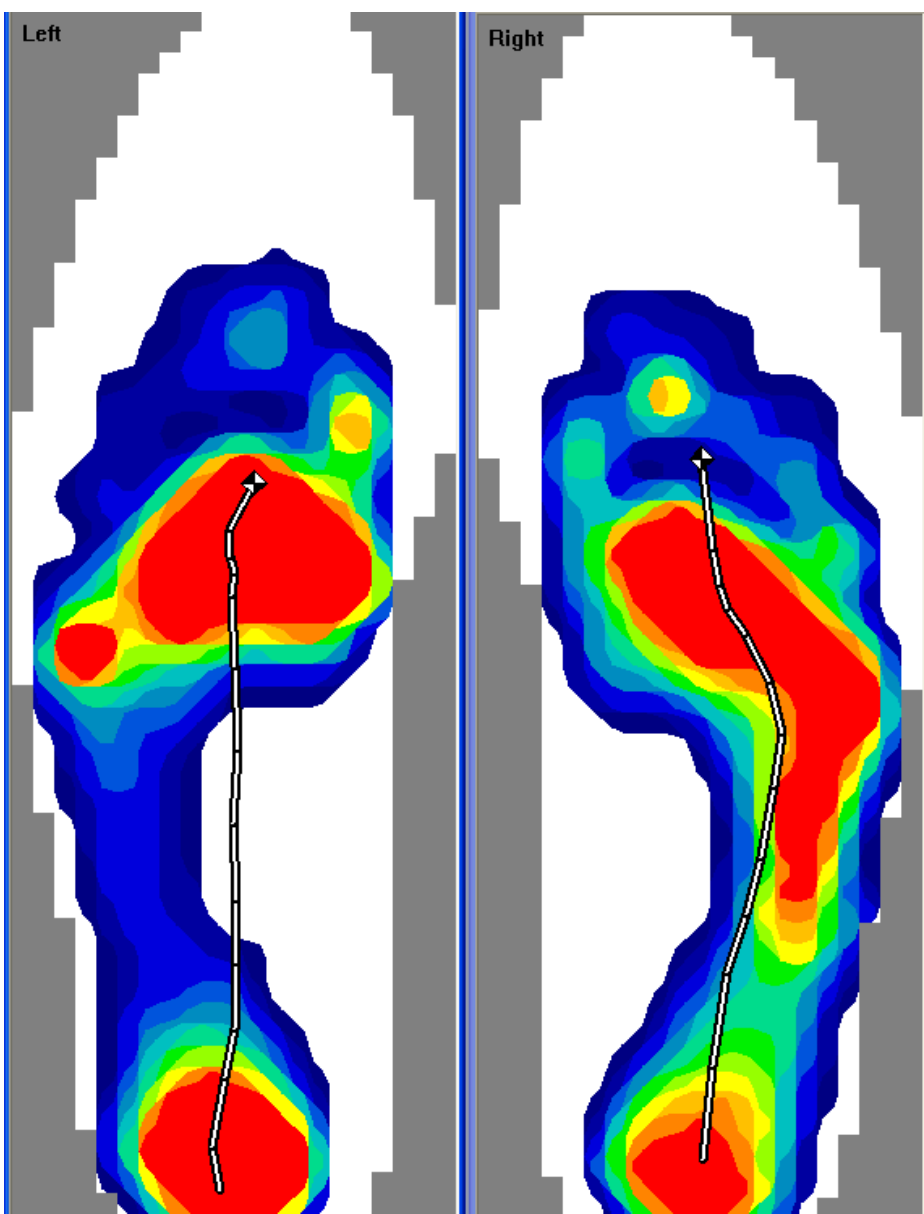

Figure 6 Average peak stance data with center of force trajectory. The patient was 17 months post hemiarthroplasty.

The poor outcome using FAOS might explain why the first metatarsal pressure was always higher in the arthrodesis group. It seems that patients felt sufficiently pain free and confident enough to take pressure through this area of the foot. Our results on the arthrodesis patients concur with DeFrino, et al., ${ }^{6}$ who also measured plantar pressures on 10 feet, to show that first MTPJ arthrodesis produced a restoration of the weight bearing function of the first ray with greater maximum force carried by the distal hallux at toe-off.

There are few comparative studies in the literature and none involving the NorthStar HemiCAP. This is the first data presented for this implant. Gibson \& Thompson ${ }^{9}$ compared a Biomet unconstrained total arthroplasty to arthrodesis. 


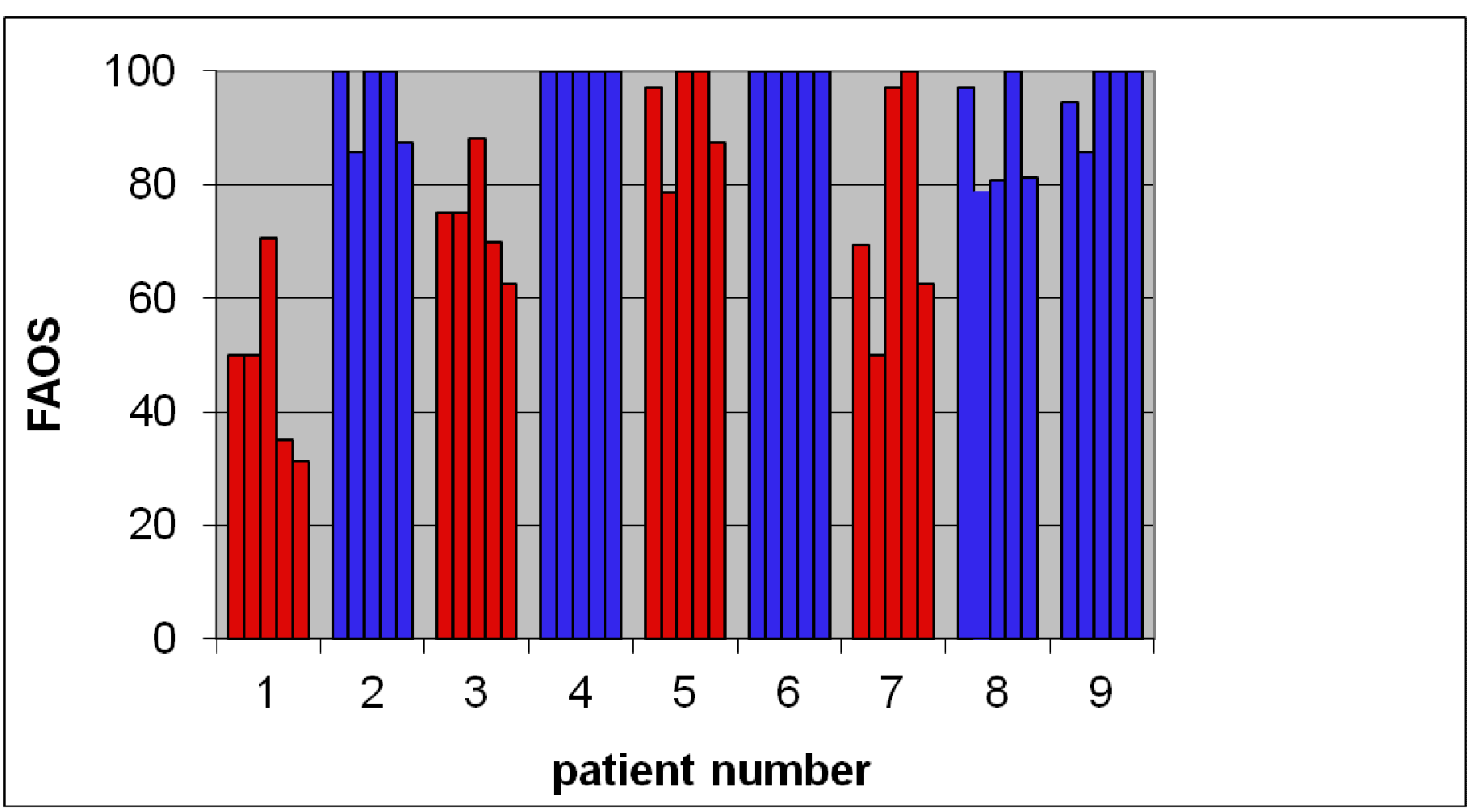

Figure 7 Foot and Ankle Outcome Score (FAOS). Red $=$ hemiarthroplasty, Blue $=$ arthrodesis, $100=$ normal FAOS score

Like these researchers, we found that the arthrodesis group had better pain scores at 24 months, although we used a FAOS rather than the VAS. Their gait analysis showed that patients in both groups tended to weight bear on the lateral border of the foot, whereas our plantar pressure and kinetic data showed a tendency for lateral foot pressure to be greater in the arthroplasty patients, with arthrodesis patients having increased pressure (and therefore increased weight bearing) under the medial foot and $1^{\text {st }}$ MTPJ.

\section{Limitations}

This study had some shortcomings. Patients were assessed retrospectively rather than prospectively, which may have affected preoperative scoring as it was based on chart review. Surgical treatment was not randomized, but was based on clinical need including patient's preference with one surgeon performing the hemiarthroplasties and arthrodeses. The sample size was limited due to the number of NorthStar HemiCAPs which were performed and could be matched to an arthrodesis procedure.
Barefoot walking was preferred to standardized footwear for kinetic and kinematic analysis. This was decided due to the difficulty in obtaining funding for such footwear. However, all plantar pressure data used the patients' own footwear in order to incorporate the insoles and this lack of standardization might be a confounding variable.

\section{Conclusion}

Arthrodesis of the $1^{\text {st }}$ MTPJ results in better FOAS scores, improved peak plantar pressure over the medial foot compared to a $1^{\text {st }}$ MTPJ hemiarthroplasty. There were minimal differences in kinetic and kinematic data. In the hemiarthroplasty patients, $1^{\text {st }}$ MTPJ ROM was considerably reduced compared to their no-operated side.

Our results of the North Star HemiCAP concur with other studies using different implants that hemiarthroplasty cannot be recommended at this time for the management of hallux rigidus. Arthrodesis remains the surgical treatment of choice. 


\section{References}

1. Coughlin MJ. Shurnas PS. Hallux rigidus. Grading and longterm results of operative treatment. JBJS 2003 85A: 2072-2088. 2. Brewster M. Does total joint replacement or arthrodesis of the first metatarsophalangeal joint yield better functional results? A systematic review of the literature. J Foot Ankle Surg 2010 49: 546-552.

3. Brodsky JW, Baum BS, Pollo FE, Mehta H. Prospective gait analysis in patients with first metatarsophalangeal joint arthrodesis for hallux rigidus. Foot Ankle Int 2007 28: 162-165. 4. Carson MC, Harrington ME, Thompson N, O'Connor JJ, Theologis TN. Kinematic analysis of a multi-segment foot model for research and clinical applications: a repeatability analysis. J Biomech 2001 34: 1299-1307.

5.Beeson P, Phillips C, Corr S, Ribbans WJ. Hallux rigidus: a cross-sectional study to evaluate clinical parameters. Foot 2009 19: 80-92.

6. DeFrino PF, Brodsky JW, Pollo FE, Crenshaw SJ, Beischer AD.

First metatarsophalangeal arthrodesis: a clinical, pedobarographic and gait analysis study. Foot Ankle Int 2002 23: 496-502.

7. Fitzgerald JA. Wilkinson JM. Arthrodesis of the metatarsophalangeal joint of the great toe. Clin Orthop Rel Res 1981, 70-77.

8. Giannini S, Vannini F, Bevoni R, Francesconi D.

Hallux Rigidus: Arthroplasty or not?

G. Bentley (ed.), European Instructional Lectures.

European Instructional Course Lectures 9, pages 239-246.

9. Gibson JN. Thomson CE. Arthrodesis or total replacement arthroplasty for hallux rigidus: a randomized controlled trial. Foot Ankle Int 2005 26: 680-690.

10. Kitaoka HB, Holiday AD, Jr., Chao EY, Cahalan TD.

Salvage of failed first metatarsophalangeal joint implant arthroplasty by implant removal and synovectomy: clinical and biomechanical evaluation. Foot Ankle 1992 13: 243-250. 11. Mann RA, Coughlin MJ, DuVries HL. Hallux rigidus: A review of the literature and a method of treatment.

Clin Orthop Rel Res 1979 57-63.

12. Olms K. Dietze A. Replacement arthroplasty for hallux rigidus. 21 patients with a 2-year follow-up. Int Orthopaedics 1999 23: 240-243.

13. Raikin SM, Ahmad J, Pour AE, Abidi N. Comparison of arthrodesis and metallic hemiarthroplasty of the hallux metatarsophalangeal joint. JBJS 2007; 89: 1979-

1985.

14. Roos EM, Brandsson S, Karlsson J. Validation of the foot and ankle outcome score for ankle ligament reconstruction. Foot Ankle Int 2001 22: 788-794.

15. Vanore JV, Christensen JC, Kravitz SR, Schuberth JM, Thomas JL, Weil LS, Zlotoff HJ, Mendicino RW, Couture SD. Diagnosis and treatment of first metatarsophalangeal joint disorders. Section 2: Hallux rigidus. J Foot Ankle Surg 2003 42: 124-136. 